\title{
The Potential of Plasminogen Activator Inhibitor-1 as Prognostic Factor of Advanced Breast Cancer
}

\author{
Erwin Danil Yulian ${ }^{1 \star}$, Umar Suratinojo ${ }^{1}$, Diani Kartini ${ }^{1}$, Samuel Johny Haryono ${ }^{2}$ and Kevin Varian Marcevianto ${ }^{3}$
}

${ }^{1}$ Division of Surgical Oncology, Department of Surgery, Faculty of Medicine, Universitas Indonesia, Dr. Cipto Mangunkusumo General Hospital, Jakarta, Indonesia.

*Corresponding author

Erwin Danil Yulian, Division of Surgical Oncology, Department of Surgery, Faculty of Medicine, Universitas Indonesia, Dr. Cipto Mangunkusumo General Hospital, Jakarta, Indonesia

${ }^{2}$ Division of Surgical Oncology, Dharmais National Cancer Center Hospital, Jakarta, Indonesia.

${ }^{3}$ Faculty of Medicine, Universitas Indonesia, Jakarta, Indonesia.
Submitted: 20 Apr 2020; Accepted: 26 Apr 2020; Published: 08 May 2020

\begin{abstract}
Background: Advanced breast cancer causes problems in morbidity, mortality, quality of life, and low survival rate. Hence, a biomarker to predict the progression of cancer is needed. Plasminogen activator inhibitor-1 (PAI-1) has been known as one of the prognostic factors. However, in vivo studies indicated that PAI-1 has controversial roles. Whether PAI-1 suppresses or promotes the development of cancer, is still being the question. The study aims to examine the role of PAI-1 in predicting the survival rate and its association with clinicopathologic factors in advanced breast cancer.
\end{abstract}

Materials and Methods: The historical cohort analytic method in advanced breast cancer patients was conducted at Dharmais National Cancer Center Hospital. Clinical data were obtained from patients' medical records. The expression of PAI-1 was assessed through immunohistochemistry assay staining of breast cancer tissue using antibody PAI-1 Santa Cruz Biotechnology, Inc., PAI-1 C-9 sc5297. Survival analysis was done to obtain the prognostic data. Moreover, its association with clinicopathologic factors was analyzed.

Results: Fifty-eight subjects were included in this study. There was a significant association between the expression of PAI-1 and survival rate (hazard ratio $[H R]=4.08,95 \%$ confidence interval $[C I]=1.75-9.50, p=0.001$ ). The Kaplan-Meier method indicated significant differences in survival rate between subjects with high expression of PAI1 and those with low expression in advanced breast cancer $(p=0.001)$. PAI-1 expression had a sensitivity of $84.7 \%$ and specificity of $60 \%$ based on immunohistochemical score cut off of 90. Furthermore, the expression of PAI-1 showed no significant association with clinicopathological factors except for histopathology grade. (Relative risk $[R R]=1.5,95 \%, C I=1.2-1.8, p=0.047)$.

Conclusion: Advanced breast cancer patients with high expression of PAI-1 have better survival. PAI-1 expression is not associated with clinicopathological factors, except for the histopathological grade.

Keywords: PAI-1, Advanced Breast Cancer, Prognostic Factor

\section{Introduction}

Currently, breast cancer is still the leading cause of death from cancer in women $(522,000$ deaths in 2012) [1]. In addition, this is the most common cancer diagnosed in women in 140 of 184 countries worldwide [1]. It was estimated that the incidence of breast cancer had increased by more than $20 \%$ since 2008 , while deaths had increased by $14 \%$. Eighty-seven percent of breast cancer cases in Indonesia were found as advanced stages, especially stage IIIB was $40 \%$ [2,3]. Although the history of treating breast cancer has experienced a long journey ranging from surgery to multimodality, what have always been the problems are the emergence of recurrence and distant metastases with various morbidity and mortality problems. Most causes of deaths in breast cancer cases are not caused by its primary tumors but caused by the metastases in secondary organs [4]. To estimate the progression of cancer, a biomarker is needed as a marker of prognostic factor.

Plasminogen activator system, in this case, plasminogen activator inhibitor (PAI-1) has been recognized as a biomarker in breast cancer $[5,6]$. In vivo studies shows that the PAI-1 role has been the subject of controversy. On one hand, PAI-1 suppresses cancer development by angiogenesis blockage, but on the other hand, it also promotes the development of cancer through angiogenesis increment and also apoptosis blockage in other studies $[6,7,8]$. The plasminogen activator system has been recognized as a breast cancer important regulator of progression. It is involved in the process of proliferation, invasion, migration of the tumor cell [6]. However, PAI-1 is found to be able to stimulate endothelial cells migration from high extracellular matrix (ECM) protein vitronectin level of perivascular area to fibronectinrich sites [6]. Besides, PAI-1 maintain the ECM level in excessive degradation, which provides a scaffold for the migration of endothelial cell and capillaries formation [6]. 
For this reason, the authors aimed to examine the role of PAI-1 as a prognostic factor and its association with clinicopathological factors in advanced breast cancer. The authors hypothesized that excessive expression of PAI-1 is a good prognosis marker and associated with clinicopathological factors in patients with advanced breast cancer.

\section{Materials and methods \\ Study design and subjects}

The study design was a retrospective analytic cohort. Moreover, the consecutive sampling method was conducted. Data collection was done by taking data from medical records for survival analysis and prognostic factors. This study had been approved by the Ethical Committee of the Dharmais National Cancer Center Hospital Number: 066/KEPK/IX/2016. The total of the minimal subjects was 46 patients, calculated based on the formula with the considerations of $80 \%$ power and an estimated $10 \%$ dropout.

Subjects of this study included patients diagnosed with stage IIIB and IV breast cancer. Histopathology data was derived from the Department of Pathological Anatomy. Diagnosis should be made from January 2011 to August 2016. Follow up was determined for 3 years, ranging from the time of diagnosis to the end of the study period in August 2016. The subjects had not got any systemic therapy when paraffin samples were taken.

Recorded clinical data consisted of age, the tumor, node, and metastases (TNM) staging, metastases presence, the type of therapy given, survival rate, and the pathological anatomy registration number. Based on the pathological anatomy registry, data regarding histopathological diagnosis, grade, estrogen and progesterone receptor (ER and PR), Ki67, human epidermal growth factor receptor (HER-2)/neu receptor, and luminal subtype were listed.

\section{Immunohistochemistry Methods}

The immunohistochemistry (IHC) was examined for PAI-1 expression on paraffin blocks from tumor tissue specimens in the pathology laboratory. Assessments of sample eligibility for PAI1 staining and histopathological examination parameters were assessed by an independent pathologist. PAI-1 antibody reagent (Santa Cruz Biotechnology, Inc. PAI-1 (C-9): sc-5297) was used in this study. This reagent was a mouse monoclonal antibody. The antibody was made against amino acids 24-158 of human origin PAI-1 with a 1:50 dilution. The immunohistochemistry examination procedure for PAI-1 was as follows: On a $4 \mu \mathrm{m}$ thick paraffin block, each specimen was blended on a water bath. It was placed on a slide and left for one night at room temperature. Thereafter, the slide was heated on a hotplate for an hour at a temperature of $600^{\circ} \mathrm{C}$. The existing sample slide was arranged on the Ventana XT device for the staining process in three hours. In the middle of the process, PAI-1 primary antibody (Santa Cruz Biotechnology, inc. PAI-1 (C-9): sc-5297) drops were added. The slide was then washed with water. Thereafter, the slide was dehydrated by using alcohol with the concentration gradation of $70 \%, 100 \%, 100 \%, 100 \%$. The slide was cleared with xylol two times. The final step was mounting them. The slide was closed with a lid and glass cover. Negative controls were made for each case without any primary antibody drop addition. Immunohistochemical staining was read out by Pathologists in the Pathological Anatomy Department. PAI-1 staining level was evaluated from the cytoplasm, stroma, and fibroblasts. Measurement was done on 100x and 400x magnification. Each result was finally calculated as an immunohistochemical score with the following formula: $\sum$ (\% positive cells) $\mathrm{x}$ (staining score) x 100 portions of positive cell counts [9].

Staining score included: $0=$ negative, $+1=$ weak, $+2=$ strong [8] . Moreover, the classification of PAI-1 staining level assessment consisted of $0 \%$ (negative), $0-10 \%$ (weakly positive), and more than $10 \%$ (strongly positive) [9].

\section{Statistical Analysis}

Statistical analysis included univariable and multivariable analysis. Survival analysis was done by making a Kaplan-Meier graph with the log-rank test. To determine the significance of risk factors, a Cox regression test statistical analysis was conducted. Then, a receiver operating characteristic ROC curve was constructed for determining the prediction of survival and area under curve (AUC). Moreover, a chi-square test was done to analyze the association between PAI-1 expression and clinicopathological factors. $P$-values of less than 0.05 were regarded to be statistically significant.

\section{Results}

Clinical characteristics of advanced breast cancer subjects Eighty-six subjects in total were obtained from January 2011 to August 2016. Fifty-eight subjects met the study inclusion, meanwhile, the remaining 28 subjects were excluded due to the condition of the damaged paraffin block. The average of follow up duration was 710 days. Most of the subjects in this study were at age $\geq 45$ years $(58.6 \%)$ with an average age of 48.10 years and a standard deviation of 10.07. Based on the TNM Staging, stage IIIB was obtained more than stage IV, which was 32 people (55.2\%). The most common histopathological type was no special type (NST), which was $68.9 \%$ (40 subjects). Based on the histopathology grading assessment, the high-grade samples were obtained more than the low grade, which were 49 people $(84.5 \%)$. There were more cases with positive hormonal receptors than the negative ones. The positive hormonal receptors included 41 positive estrogen receptors (ER) $(70.4 \%)$ and 40 positive progesterone receptors $(\mathrm{PR})(69.0 \%)$. Subjects with negative Her2 were 44 people $(75.9 \%)$. Based on the intrinsic subtype grouping, there were 44 people $(75.9 \%)$ of luminal a groups. Based on the type of therapy, 38 subjects $(63.8 \%)$ received chemotherapy, 36 subjects $(50 \%)$ received hormonal therapy, and 47 subjects $(81 \%)$ undergone mastectomies. Metastases occurred in 26 subjects (44.8\%). Subjects with PAI-1 expression $\geq 90$ cut off were 41 people $(70.7 \%)$. However, subjects with PAI-1 expression below 90 were 17 people $(29.3 \%)$. Based on this study, the number of subjects who lived until the end of the three years follow-up was 35 people $(60.3 \%)$, meanwhile, 23 people (39.7\%) died.

\section{PAI-1 Immunohistochemical Staining Results}

Immunohistochemical staining illustrated that the primary tumor examined had a variation in the intensity and presentation of the amount of the colored cell based on PAI-1 expression. Browncolored PAI-1 was mainly detected more in the cytoplasm and stroma than in fibroblasts. Some of the results of the PAI-1 immunohistochemical staining can be seen in figure 1 


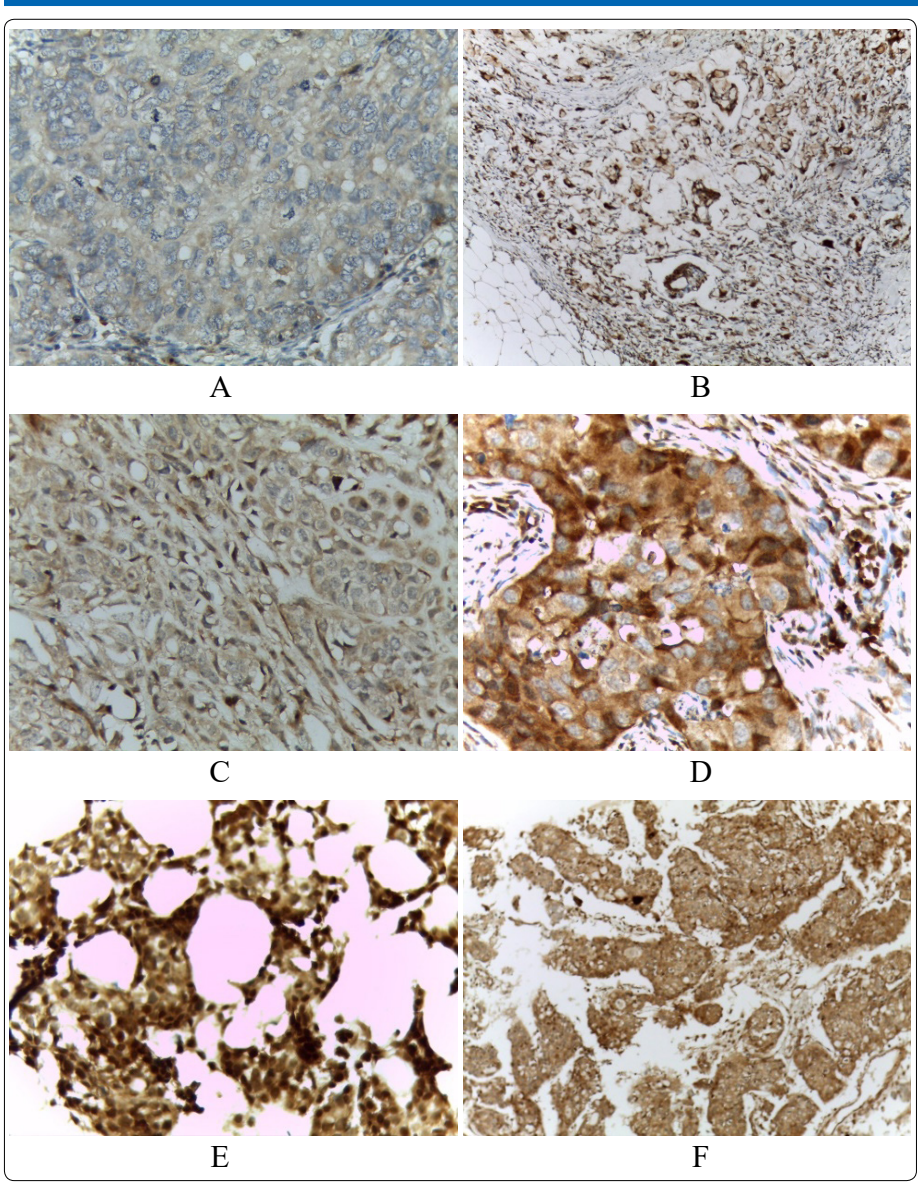

Figure 1: Example of PAI-1 detection in IHC staining results. (A) Negative result on 100x magnification. (B) +1 staining score with $75 \%$ positive cells on $100 x$ magnification. (C) +1 staining score with $90 \%$ positive cells on $100 x$ magnification. (D) +2 staining score with $80 \%$ positive cells on $400 x$ magnification. (E) +2 staining score with $100 \%$ positive cells on $400 x$ magnification. $(\mathrm{F})$ +2 staining score with $100 \%$ positive cells on $100 x$ magnification.

\section{Analysis of PAI-1 with prognosis in advanced breast cancer subjects}

Based on the univariable analysis, there was a significant association between PAI-1 expression and survival rate $(\mathrm{HR}=4.08,95 \% \mathrm{CI}$ $=1.75-9.50, p=0.001)$ using the log-rank test. Thirty subjects
(73.2\%) with PAI-1>90 survived, whereas 11 people $(26.8 \%)$ died. However, five subjects $(29.4 \%)$ with PAI-1 $<90$ survived, whereas 12 people $(70.6 \%)$ died. Kaplan-Meier analysis of survival in Figure 1A showed a significant difference between the PAI-1 expression groups on the survival rate of advanced breast cancer cases ( $p=0.001)$, where the high PAI-1 group had longer survival. High PAI-1 group had a long life span of 1408 days, while the low PAI-1 group had a life span of 540 days. Using the ROC curves (Figure 2B), it was found that the value of the AUC was $66.7 \%$ $(95 \% \mathrm{CI}=51.4-82.2, p=0.033)$. This showed that the percentage of PAI-1 cells in primary advanced breast cancer tissue could be used as a predictive factor for survival rate. The intersection point between the sensitivity and specificity graphs was set with the cutoff point value is 90 . This resulted in a sensitivity of $84.7 \%$ and a specificity of $60 \%$.

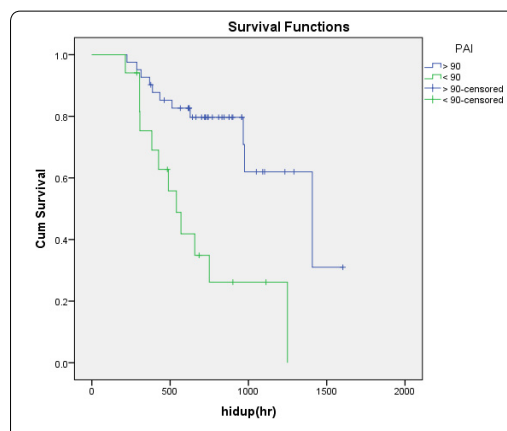

A

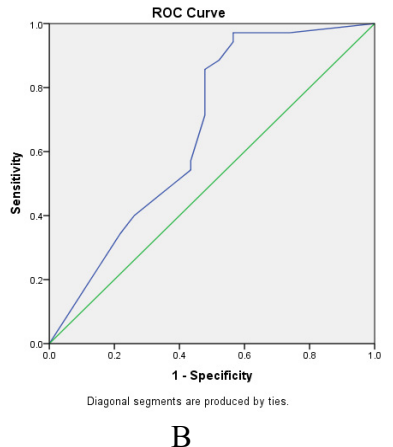

B
Figure 2: Performance of the PAI-1 biomarker as the prognostic factor. (A) The Kaplan-Meier survival curve of overall survival of subjects with advanced breast cancer using the PAI-1 expression. (B) ROC Curve analysis of the PAI-1 biomarker expression.

The association between all variables and the survival rate was also observed as seen in table 1 . Based on this table, it can be seen that grading, luminal A, and hormonal therapy factors have a significant hazard ratio. Each variable with a $p$-value $<0.25$ from the univariate analysis was then included in the multivariate analysis. Those variables were grading, hormonal therapy, luminal A, stage and PAI-1 expression. The Cox Regression Test with the stepwise method was conducted by inserting five variables. It turned out that there was collinearity between grading variable and PAI-1 expression. Hence, the grade variable was excluded. After the separation, there were changes in the hazard ratio of PAI-1 expression, luminal, and staging variables as listed in table 1.

Table 1: Univariable and multivariable analysis of survival rate

\begin{tabular}{|c|c|c|c|c|c|c|}
\hline \multirow[t]{2}{*}{ Variable } & \multicolumn{3}{|c|}{ Univariate Analysis } & \multicolumn{3}{|c|}{ Multivariate Analysis } \\
\hline & \begin{tabular}{|l|} 
OR \\
\end{tabular} & $95 \% \mathrm{CI}$ & $p$-value & Adjusted HR & $95 \%$ CI & $p$-value \\
\hline Age (years) & 1.06 & $0.69-1.61$ & $0.778^{\mathrm{a}}$ & & & \\
\hline TNM Staging & 1.30 & $0.84-2.02$ & $0.217^{\mathrm{a}}$ & 2.883 & $1.054-7.885$ & $0.039^{\mathrm{c}}$ \\
\hline Histopathological Grade & 1.80 & $1.42-2.41$ & $0.008^{\mathrm{b}}$ & & & \\
\hline Metastases & 1.22 & $0.79-1.88$ & $0.362^{\mathrm{a}}$ & & & \\
\hline Luminal A & 0.65 & $0.34-1.23$ & $0.125^{\mathrm{a}}$ & 3.044 & $1.058-8.753$ & $0.039^{\mathrm{c}}$ \\
\hline Hormonal therapy & 0.65 & $0.39-1.08$ & $0.070^{\mathrm{a}}$ & & & \\
\hline Chemotherapy & 1.27 & 0.84-1.89 & $0.275^{\mathrm{a}}$ & & & \\
\hline Mastectomy & 1.06 & $0.64-1.77$ & $0.804^{\mathrm{a}}$ & & & \\
\hline Histopathological type & 1.10 & $0.60-1.80$ & $0.617^{\mathrm{a}}$ & & & \\
\hline PAI-1 & $2.28^{* *}$ & $1.16-5.31$ & $0.003^{\mathrm{a}}$ & 3.833 & $1.643-8.939$ & $0.002^{\mathrm{c}}$ \\
\hline
\end{tabular}


* $p$-value a came from chi-square tests

${ }^{*} p$-value ${ }^{\mathrm{b}}$ came from Fisher's Exact Test

${ }^{*} p$-value ${ }^{c}$ came from Cox Regression Test stepwise method without considering grade variables due to collinearity between PAI-1 and grade variables

**using RR value

\section{Analysis between PAI-1 expression and clinicopathology factors}

In general, none of the association between PAI-1 expression and each clinicopathological factors was statistically significant, except for histopathology grade variable with the relative risk of $1.5(p=0.047)$. The detailed data of the analysis can be observed in Table 2.

Table 2: Association between PAI-1 expression and clinicopathology factors

\begin{tabular}{|c|c|c|c|c|c|c|}
\hline \multirow{3}{*}{\multicolumn{2}{|c|}{ Variable }} & \multicolumn{4}{|c|}{ PAI-1 } & \multirow{3}{*}{$\begin{array}{l}p \text {-value } \\
\text { (Chi-square } \\
\text { test) }\end{array}$} \\
\hline & & \multicolumn{2}{|c|}{$\geq 90$} & \multicolumn{2}{|c|}{$<90$} & \\
\hline & & $\mathbf{n}$ & $\%$ & n & $\%$ & \\
\hline \multirow{2}{*}{ Age (years) } & $<45$ & 17 & 70.8 & 7 & 29.2 & \multirow{2}{*}{$0.989^{\mathrm{a}}$} \\
\hline & $\geq 45$ & 24 & 70.6 & 10 & 29.4 & \\
\hline \multirow{2}{*}{ TNM Staging } & $3 \mathrm{~B}$ & 23 & 74.2 & 8 & 25.8 & \multirow{2}{*}{$0.530^{\mathrm{a}}$} \\
\hline & 4 & 18 & 66.7 & 9 & 33.3 & \\
\hline \multirow{2}{*}{ Histopathological Grade } & Low & 9 & 100.0 & 0 & 0.0 & \multirow{2}{*}{$0.047^{\mathrm{b}}$} \\
\hline & High & 32 & 65.3 & 17 & 34.6 & \\
\hline \multirow{2}{*}{ ER } & Negative & 13 & 76.5 & 4 & 23.5 & \multirow{2}{*}{$0.533^{\mathrm{a}}$} \\
\hline & Positive & 28 & 68.3 & 13 & 31.7 & \\
\hline \multirow{2}{*}{ PR } & Negative & 11 & 61.1 & 7 & 38.9 & \multirow{2}{*}{$0.282^{\mathrm{a}}$} \\
\hline & Positive & 30 & 75.0 & 10 & 25.0 & \\
\hline \multirow{2}{*}{ Her2 } & Negative & 32 & 72.7 & 12 & 27.3 & \multirow{2}{*}{$0.596^{\mathrm{a}}$} \\
\hline & Positive & 9 & 64.3 & 5 & 35.7 & \\
\hline \multirow{2}{*}{ Ki67 } & $<14 \%$ & 15 & 83.3 & 3 & 16.7 & \multirow{2}{*}{$0.156^{\mathrm{a}}$} \\
\hline & $\geq 14 \%$ & 26 & 65.0 & 14 & 35.0 & \\
\hline \multirow{2}{*}{ Metastases } & No & 23 & 71.9 & 9 & 28.1 & \multirow{2}{*}{$0.826^{\mathrm{a}}$} \\
\hline & Yes & 18 & 69.2 & 8 & 30.8 & \\
\hline \multirow{2}{*}{ Hormonal therapy } & No & 15 & 68.2 & 7 & 31.8 & \multirow{2}{*}{$0.743^{a}$} \\
\hline & Yes & 26 & 72.2 & 10 & 27.8 & \\
\hline \multirow{2}{*}{ Mastectomy } & No & 9 & 81.8 & 2 & 18.2 & \multirow{2}{*}{$0.368^{\mathrm{a}}$} \\
\hline & Yes & 32 & 68.1 & 15 & 31.9 & \\
\hline \multirow{2}{*}{ Chemotherapy } & No & 14 & 70.0 & 6 & 30.0 & \multirow{2}{*}{$0.933^{\mathrm{a}}$} \\
\hline & Yes & 27 & 71.1 & 11 & 28.9 & \\
\hline \multirow[t]{2}{*}{ Histopathological type } & NST & 27 & 67.5 & 13 & 32.5 & \multirow{2}{*}{$0.426^{\mathrm{a}}$} \\
\hline & Non-NST & 14 & 77.8 & 4 & 22.2 & \\
\hline \multirow{2}{*}{ Luminal A } & No & 10 & 71.4 & 4 & 28.6 & \multirow{2}{*}{$0.944^{\mathrm{a}}$} \\
\hline & Yes & 31 & 70.5 & 13 & 29.5 & \\
\hline
\end{tabular}

${ }^{*} p$-value a came from chi-square tests

${ }^{*} p$-value ${ }^{\mathrm{b}}$ came from Fisher's Exact Test

\section{Discussion}

This study proves that high PAI-1 expression has a better survival rate than low PAI-1 significantly $(p=0.001)$. Subjects with high PAI-1 expression will be able to survive 4.08x longer than those with low PAI-1. Based on the Kaplan-Meier curve, it was found that subjects with high PAI- 1 had a higher survival rate of 868 days more than subjects with low PAI-1. Moreover, with 90 cutoffs, the sensitivity obtained is $84.7 \%$ and specificity is $60 \%$. This specificity value was caused by the overlap of the PAI-1 values to distinguish between died and survived subjects.

The result of this study differs from some previous studies $[6,10]$. However, the previous studies evaluates the role of PAI1 expression in early-stage breast cancer $[6,11]$. The difference between this study and the others might be due to the aim of this study, which was designed to analyze the PAI-1 as a prognostic factor in advanced breast cancer (including stadium IIIB and IV) which lead to different characteristics. The dual role of PAI-1 is still a contradictory matter for the progression of each breast cancer [12]. First, PAI-1 could be involved in tumor growth, invasion, and metastases of breast cancer. PAI-1 has a role in maintaining the amount of ECM needed for cancer cells during the migration or regulating the pericellular non-proteolytic function of the uPA system during cell migration [12]. Moreover, a study by Knoop A, et al. shows PAI-1 as an independent factor in predicting distant metastases [13]. Second, the expression of PAI-1 in tumors can be a defense mechanism of normal tissue against cancer cells. These are imposed by the formation of plasmin and capable to counteract the tumor growth and invasion [11]. PAI-1 can influence cell surface expression and internalization of the uPAuPAR complex which causes inhibition of invasion and metastasis. Moreover, the discrepancy could be attributed to the complexity of the interactions between proteinases and their inhibitors in the development of breast cancer, which should also be analyzed [14].

Based on the analysis of the association between PAI-1 expression and clinicopathological factors, PAI-1 expression did not affect any clinicopathological factors, except for the histopathology grade. Nevertheless, it can be seen that there is a high expression of PAI-1 on all clinicopathological factors. The study by Bouchet, et al. conveys that PAI-I level is associated with hormonal status [15]. High levels of PAI-1 were found mainly in postmenopausal women. Decreased estrogen production is capable to affect PAI-I activity to be increased, which then reflects the degree of control of estrogen secretion of PAI-1 $[15,16]$.

Although there are differences in PAI-1 expression related to differences in the pathology of breast cancer, there are no extraordinary individual factors that are consistently related to prognosis as proposed by Bouchet, et al [15]. Therefore, other factors such as intrinsic factors (cytokines, lipids, hormones) and extrinsic factors (injury and genetic damage) may be important to take into account [17]. The balance of the relative concentration of proteinase (uPA) and its inhibitors (PAI-1) might also need to be analyzed which determines ECM degradation in tumor invasion and metastasis in vivo [18].

In conclusion, high PAI-1 expression as a prognostic factor of advanced breast cancer patients indicates a better survival rate than the low PAI-1 expression case. Moreover, PAI-1 expression in advanced breast cancer is not related to clinicopathology factors, except for histopathological grade. 


\section{References}

1. Latest world cancer statistics Global cancer burden rises to 14.1 million new cases in 2012 : Marked increase in breast cancers must be addressed. Int Agency Res Cancer, World Heal Organ (2013).

2. Setyowibowo H, Purba FD, Hunfeld JAM, Iskandarsyah A, Sadarjoen SS, et al. (2018) Quality of life and health status of Indonesian women with breast cancer symptoms before the definitive diagnosis: A comparison with Indonesian women in general. PLOS One 13: 1-11.

3. Tjindarbumi D, Ramli M, Watanabe S, Darwis I, Sakamoto $\mathrm{G}$, et al. (1995) Clinicopathological aspects of breast cancer: A joint study between Indonesia and Japan. Med J Indones 4: 148-155.

4. Scully OJ, Bay B-H, Yip G, Yu Y (2012) Breast Cancer Metastasis. Cancer Genomics Proteomics 9: 311-320.

5. Harris L, Fritsche H, Mennel R, Norton L, Ravdin P, et al. (2007) American society of clinical oncology 2007 update of recommendations for the use of tumor markers in breast cancer. J Clin Oncol 25: 5287-5312.

6. Duffy MJ, McGowan PM, Harbeck N, Thomssen C, Schmitt M (2014) UPA and PAI-1 as biomarkers in breast cancer: Validated for clinical use in level-of-evidence-1 studies. Breast Cancer Res 16: 1-10.

7. Dass K, Ahmad A, Azmi AS, Sarkar SH, Sarkar FH (2008) Evolving role of uPA/uPAR system in human cancers. Cancer Treat Rev 34: 122-136.

8. Tang L, Han X (2013) The urokinase plasminogen activator system in breast cancer invasion and metastasis. Biomed Pharmacother 67: 179-182.

9. Minisini AM, Fabbro D, Di Loreto C, Pestrin M, Russo S, et al. (2007) Markers of the uPA system and common prognostic factors in breast cancer. Am J Clin Pathol 128: 112-117.

10. Look MP, Van Putten W, Duffy M, Harbeck N, Christensen IJ, et al. (2003) Pooled analysis of prognostic impact of uPA and PAI-1 in breast cancer patients. Thromb Haemost 90: 538548.

11. Märkl B, Kazik M, Harbeck N, Jakubowicz E, Hoffmann R, et al. (2019) Impact of uPA/PAI-1 and disseminated cytokeratinpositive cells in breast cancer. BMC Cancer 19: 1-10.

12. Durand MKV, Bødker JS, Christensen A, Dupont DM, Hansen M, et al. (2004) Plasminogen activator inhibitor-I and tumour growth, invasion, and metastasis. Thromb Haemost. 92: 3546.

13. Knoop A, Andreasen PA, Andersen JA, Hansen S, Lænkholm A V, et al. (1998) Prognostic significance of urokinase-type plasminogen activator and plasminogen activator inhibitor-1 in primary breast cancer. Br J Cancer 77: 932-940.

14. Foekens JA, Peters HA, Look MP, Portengen H, Schmitt M, et al. (2000) The urokinase system of plasminogen activation and prognosis in 2780 breast cancer patients. Cancer Res 60: 636-643.
15. Bouchet C, Spyratos F, Martin PM, Hanéne K, Gentile A, et al. (1994) Prognostic value of urokinase-type plasminogen activator (uPA) and plasminogen activator inhibitors PAI-1 and PAI-2 in breast carcinomas. Br J Cancer 69: 398-405.

16. Young PL, Men N (1998) Visceral Fat Accumulation Is an Important Determinant of Modulation by Cross-Sex Hormone Administration 1: 1716-1723.

17. Lee CC, Huang TS (2005) Plasminogen Activator Inhibitor-1: The Expression, Biological Functions, and Effects on Tumorigenesis and Tumor Cell Adhesion and Migration. J Cancer Mol 1: 25-36.

18. Baker EA, Stephenson TJ, Reed MWR, Brown NJ (2002) Expression of proteinases and inhibitors in human breast cancer progression and survival. J Clin Pathol - Mol Pathol. 55: $300-304$.

Copyright: (C2020 Erwin Danil Yulian, et al. This is an open-access article distributed under the terms of the Creative Commons Attribution License, which permits unrestricted use, distribution, and reproduction in any medium, provided the original author and source are credited. 